\title{
Diffraction and the gluon mass
}

\author{
M. B. Gay Ducati \\ Department of Physics, University of Wisconsin, Madison, Wisconsin 53706 \\ and Instituto de Física, Universidade Federal do Rio Grande do Sul, \\ Avenida Bento Gonçalves, 9500, 91501-970, Porto Alegre, Rio Grande do Sul, Brazil \\ F. Halzen \\ Department of Physics, University of Wisconsin, Madison, Wisconsin 53706 \\ A. A. Natale \\ Instituto de Física Teórica, Universidade Estadual Paulista, Rua Pamplona, 145, 01405, São Paulo, São Paulo, Brazil
}

(Received 20 April 1993)

\begin{abstract}
We recently proposed a QCD Pomeron described by the exchange of two nonperturbative gluons characterized by a dynamically generated gluon mass. It is shown here that data on elastic scattering, exclusive $\rho$ production in deep inelastic scattering, and the $J / \psi$-nucleon total cross section can be successfully described in terms of a single gluon mass $m_{g} \simeq 0.37 \mathrm{GeV}$. We observe that the total cross sections of hadrons with small radii, such as $J / \psi$, have a marked dependence on the effective gluon mass.

PACS number(s): 12.38.Lg, 12.38.Qk, 12.40.Gg, 13.85.Dz
\end{abstract}

\section{INTRODUCTION}

Diffractive phenomena are described by the exchange of the Pomeron. In the framework of QCD the Pomeron is understood as the exchange of two (or more) gluons [1]. Because the exchange of two perturbative gluons cannot reproduce the experimental results, the Pomeron is constructed using two nonperturbative gluons whose properties are dictated by the expected structure of the QCD vacuum. This was discussed by Landshoff and Nachtmann [2] (LN). The nonperturbative gluons should not propagate over long distances; i.e., there is a finite correlation length for the gluon field in the vacuum which can be understood in terms of gluon condensates [2]. It is interesting to note that in his original paper Low [1] made use of a gluon mass to simulate the confinement property of QCD. It is clear, however, that a bare mass cannot be introduced in QCD. The correlation length quoted in Refs. $[1,2]$ should be determined from first principles.

Recently we proposed a QCD Pomeron model of this type using a solution of the Schwinger-Dyson equation for the gluon propagators which contains a dynamically generated gluon mass [3]. We showed that such a model describes elastic proton-proton scattering [4]. This solution, unlike other nonperturbative propagators [5], is obtained in a gauge invariant way [3] and satisfies one of the basic constraints on the nonperturbative Pomeron model [2], namely that it should be finite at $k^{2}=0$. A relation between the Pomeron and an effective gluon mass was obtained in Ref. [4] which is consistent with analytical [3] and lattice [6] calculations.

The phenomenology of this model is very rich. In this paper we show how it successfully accommodates the data on $\rho$ meson production in deep inelastic scattering and the $J / \psi$-nucleon total cross section in terms of the same gluon mass derived from elastic scattering. It has been realized for some time that exclusive $\rho$ meson production in deep inelastic scattering is an interesting laboratory for studying the nonperturbative Pomeron (i.e., the gluon mass) $[7,8]$. Our results will illustrate that also the $J / \psi$-nucleon total cross section [9] exhibits an especially strong dependence on the dynamical gluon mass. The existence of a massive gluon propagator has deep phenomenological implications [10], and we here obtain an independent determination of the gluon mass within the context of a fully consistent description of diffractive scattering.

\section{LN MODEL AND THE QUARK-POMERON COUPLING}

In the LN model Pomeron exchange between quarks has a structure similar to a photon exchange diagram with the amplitude

$$
i \beta_{0}^{2}\left(\bar{u} \gamma_{\mu} u\right)\left(\bar{u} \gamma^{\mu} u\right)
$$

where $\beta_{0}$ is the strength of the Pomeron coupling to quarks:

$$
\beta_{0}^{2}=\frac{1}{36 \pi^{2}} \int d^{2} k\left[g^{2} D\left(k^{2}\right)\right]^{2},
$$

where $g^{2} / 4 \pi$ is the strength of the nonperturbative coupling. Its experimental value is $\beta_{0}^{2}=4 \mathrm{GeV}^{-2}$ [4]. Notice that for an infrared-finite propagator the integral in Eq. (2) converges. This convergence is required in order to reproduce the additive quark rule for total cross sections and to understand the different coupling of the Pomeron to heavy quarks, as discussed in detail by Landshoff and Nachtmann [2]. In the following we will use a massive gluonic propagator to compute $\beta_{0}^{2}$.

The possibility that the gluon has a dynamically gen- 
erated mass has been advocated by Cornwall in a series of papers $[3,11]$. He has constructed a gauge invariant truncation of the gluonic Schwinger-Dyson equation whose solution, through expansion or numerical techniques, is shown to be massive. More importantly, the numerical solution is well fitted by a trial function which exhibits the correct renormalization group behavior for the gluon propagator. In the Feynman gauge this solution is given by $D_{\mu \nu}=-i g_{\mu \nu} D\left(k^{2}\right)$, where, in Euclidean space, [3]

$D^{-1}\left(k^{2}\right)=\left[k^{2}+m^{2}\left(k^{2}\right)\right] b g^{2} \ln \left[\frac{k^{2}+4 m^{2}\left(k^{2}\right)}{\Lambda^{2}}\right]$

with the momentum-dependent dynamical mass given by $[12]$

$$
m^{2}\left(k^{2}\right)=m_{g}^{2}\left[\frac{\ln \left(\frac{k^{2}+4 m_{g}^{2}}{\Lambda^{2}}\right)}{\ln \frac{4 m_{g}^{2}}{\Lambda^{2}}}\right]^{-12 / 11}
$$

In the above equations $m_{g}$ is the gluon mass, and $b=$ $\left(33-2 n_{f}\right) / 48 \pi^{2}$ is the leading order coefficient of the $\beta$ function of the renormalization group equation, where $n_{f}$ is the number of flavors taken as 3. We are including the effect of fermion loops in $b$. The coupling $g$ is frozen and therefore $g^{2} D\left(k^{2}\right)$ is formally independent of $g$. Consistency with the numerical solution requires that $g$ be in the range $1.5-2$. The solution is valid only for $m_{g}>\Lambda / 2[3]$. By relating the gluon mass to the gluon condensate, Cornwall estimated that $m_{g}=0.5 \pm 0.2 \mathrm{GeV}$ for $\Lambda_{\mathrm{QCD}}=0.3 \mathrm{GeV}$. This constraint combined with the value of the Pomeron coupling to quarks, see Eq. (2), fixes the nonperturbative propagator $[2,7]$. Figure 1 shows the determination of $\beta_{0}^{2}$ as a function of the gluon mass using Eq. (3) for three different values of $\Lambda$. For $\Lambda=300 \mathrm{MeV}$ and $m_{g}=380 \mathrm{MeV}$ we obtain $\beta_{0} \simeq 2 \mathrm{GeV}^{-1}$. This value of the gluon mass is consistent with $m_{g}=370 \mathrm{MeV}$ obtained from fits to the $p p$ total cross section. We find that a gluon mass $m_{g}=(1.2-2) \Lambda_{\mathrm{QCD}}$ is in agreement with experiment. For easy comparison with previous works $[4,3]$

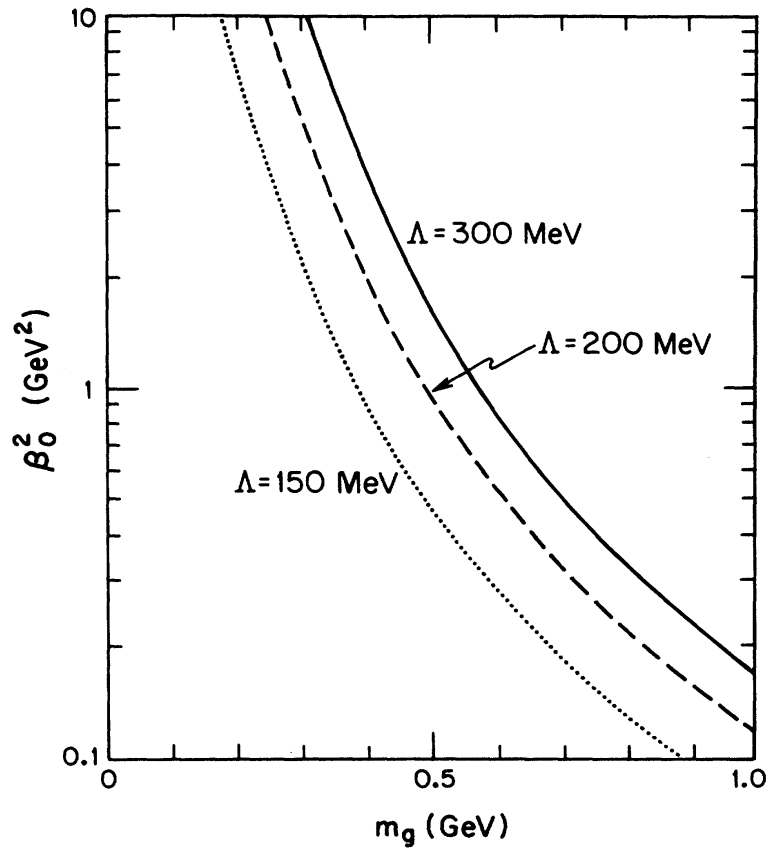

FIG. 1. Pomeron coupling to quarks $\left(\beta_{0}^{2}\right)$ as a function of the gluon mass $m_{g}$ for different values of $\Lambda: \Lambda=300 \mathrm{MeV}$ (solid curve), $\Lambda=200 \mathrm{MeV}$ (dashed curve), and $\Lambda=150 \mathrm{MeV}$ (dotted curve).

we choose $\Lambda=0.3 \mathrm{GeV}$, and will show that a variety of experimental data require a gluon mass $m_{g} \simeq 0.37 \mathrm{GeV}$.

\section{EXCLUSIVE $\rho$ PRODUCTION IN DEEP INELASTIC SCATTERING}

The Pomeron-quark coupling decreases when either or both of the quark legs in the Pomeron-quark-quark vertex move far off shell [7]. This off-shell coupling can be measured in the exclusive deep inelastic scattering (DIS) process $\gamma^{*} p \rightarrow \rho p$ [7]. The amplitude for this process is given by [8]

$$
A=i \frac{8 \sqrt{2}}{3 \pi} m_{\rho} \sqrt{\frac{\alpha_{s}^{2}\left(Q^{2}\right)}{\alpha_{n}^{2}}} P_{i} \int d k^{2} \frac{-4 k^{2}+t}{\left(m_{\rho}^{2}+Q^{2}-t\right)\left(-4 k^{2}+Q^{2}+m_{\rho}^{2}\right)}\left[4 \pi \alpha_{n} D\left(k^{2}+t / 4\right)\right]^{2}
$$

where $\alpha_{s}\left(Q^{2}\right)$ is the strong running coupling constant and $\alpha_{n}=g^{2} / 4 \pi$. Also, $q, P$, and $P^{\prime}$ are the fourmomenta of the photon and incoming and outgoing protons, $Q^{2}=-q^{2}, t=\left(P-P^{\prime}\right)^{2}$, and $m_{\rho}$ is the $\rho$ meson mass. $P_{i}$ will describe the transverse or longitudinal vector meson polarizations, with $P_{T}=\frac{1}{2} w^{2}$ and $P_{L}=$ $\frac{1}{2} w^{2}\left[\left(t+m_{\rho}^{2}+Q^{2}\right) / 2 m_{\rho} \sqrt{Q^{2}}\right]$, where $w^{2}=(P+q)^{2}$. In Ref. [8] the nonperturbative propagator is given by the ansatz

$$
\alpha_{n} D\left(k^{2}\right)=\frac{3 \beta_{0}}{\sqrt{2 \pi} \mu_{0}} \exp \left(-\frac{k^{2}}{\mu_{0}^{2}}\right)
$$

where $\mu_{0}$ is a mass scale. This function is required to match the perturbative $1 / k^{2}$ propagator at a given $Q_{0}^{2}$. This determines $Q_{0}^{2}$ and $\alpha_{n}$. In contrast the QCD motivated propagator of Eq. (4) is valid for the entire range of momentum with $g=1.5$. As the coupling $g$ is frozen at small $k^{2}$, we will assume $g^{2}\left(k^{2}\right) \simeq g^{2} /\left(1+b g^{2} \ln \frac{k^{2}+m_{g}^{2}}{\Lambda^{2}}\right)$, i.e., for $k^{2} \rightarrow 0, g^{2}\left(k^{2}\right)$ approaches the frozen value of $g$, and we recover the perturbative coupling at high momentum.

The differential cross section is given by [8]

$$
\frac{d \sigma}{d t}=\left[\frac{\alpha_{\mathrm{elm}}}{4 w^{4}}|A|^{2} \Phi^{2}\right] Z^{2}\left[3 F_{1}(t)\right]^{2},
$$


where $\alpha_{\text {elm }}$ is the electromagnetic coupling constant, $\Phi$ gives the strength of the $q \bar{q} \rho$ vertex, $F_{1}(t)$ is the proton elastic form factor,

$$
F_{1}(t)=\frac{4 m_{p}^{2}-2.79 t}{4 m_{p}^{2}-t} \frac{1}{(1-t / 0.71)^{2}}
$$

where $m_{p}$ is the proton mass and $Z$ is equal to

$$
Z=\left(\frac{w^{2}}{w_{0}^{2}}\right)^{0.08+\alpha^{\prime} t}
$$

where $w_{0}^{2} \simeq 1 / \alpha^{\prime}$ and $\alpha^{\prime}=(2 \mathrm{GeV})^{-2}$. Equation (9) introduces the Pomeron exchange dependence on energy. We must stress that the two-gluon exchange gives only the constant part of the cross section; i.e., it reproduces a Pomeron trajectory $1+0 t$. The existence of higher-order corrections in the form of a ladderlike gluon structure in the $t$ channel will give the Regge trajectory $1+0.08+\alpha^{\prime} t$. Because at $t=0$ the energy dependence is very small we can compare the value of $\beta_{0}$ calculated in the previous section directly to the experimental one.

The total cross section for $\gamma^{*} p \rightarrow \rho p$ which is the sum of the transverse and longitudinal parts $\sigma_{\text {total }}=\sigma_{T}+\epsilon \sigma_{L}$ is shown in Fig. 2 for $\Lambda=0.3 \mathrm{GeV},\langle w\rangle=12 \mathrm{GeV}$ and $\epsilon=0.85$. We conclude that $m_{g}=0.37 \mathrm{GeV}$ describes the data. Notice that there is a strong variation of the cross section with the gluon mass and that, once $\Lambda$ is fixed, this is a truly one-parameter fit. For very low values of $Q^{2}$ nonperturbative effects of the quarks involved in the process are important and the disagreement with the data is expected $[7,8]$.

\section{IV. $J / \psi$-NUCLEON SCATTERING}

Meson-nucleon scattering within the two-gluon model has been previously discussed $[9,13]$. It provides yet another test of our model. The cross section of heavy mesons strongly depends on the gluon mass. We will show that in our picture of the Pomeron the famil-

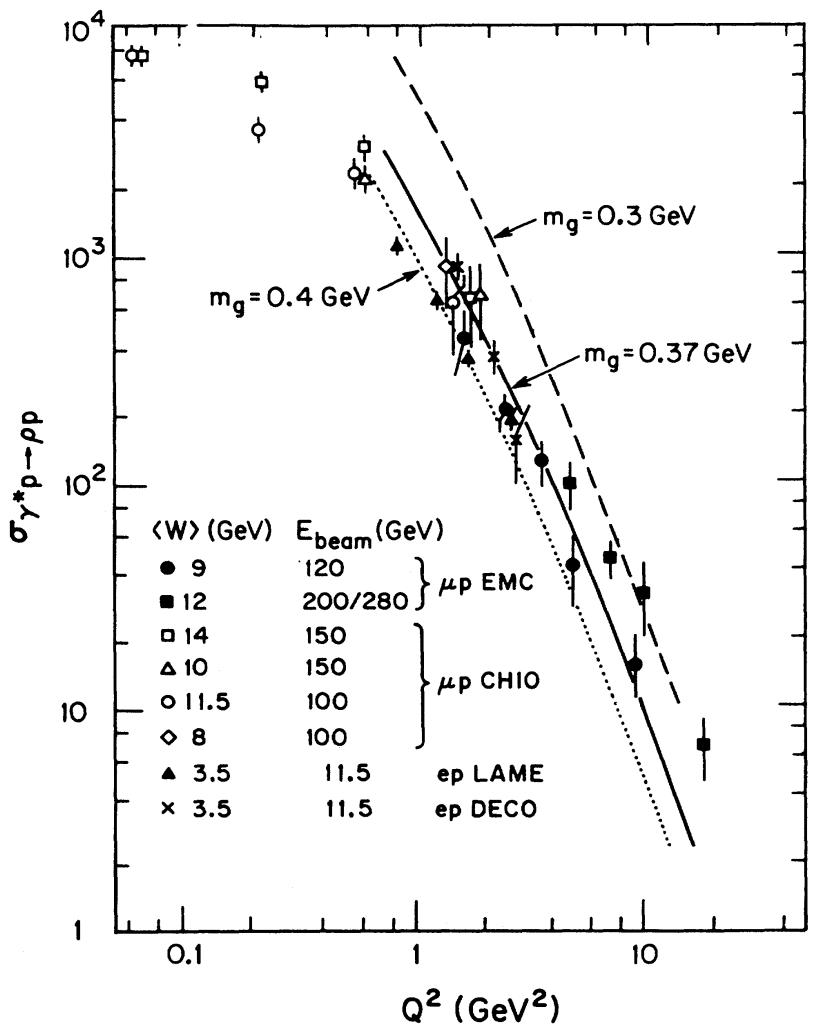

FIG. 2. Total cross section for exclusive $\rho^{0}$ production as a function of the gluon mass $m_{g}: m_{g}=0.3 \mathrm{GeV}$ (dashed curve), $m_{g}=0.37 \mathrm{GeV}$ (solid curve), and $m_{g}=0.4 \mathrm{GeV}$ (dotted curve). Data from Ref. [16].

iar relation between cross section and effective radii of hadrons [14] breaks down for heavy particles such as $J / \psi$. This can be tested experimentally.

The amplitude of meson-nucleon scattering is given by $[9,13]$

$A=i \frac{32}{9} s \alpha^{2} \int d^{2} k D\left(k^{2}\right) D\left((2 \mathbf{Q}-\mathbf{k})^{2}\right) 2\left[f_{M}\left(Q^{2}\right)-f_{M}\left((\mathbf{Q}-\mathbf{k})^{2}\right)\right] 3\left[f_{N}\left(Q^{2}\right)-f_{N}\left(Q^{2}-\frac{3}{2} \mathbf{Q} \cdot \mathbf{k}+\frac{3}{4} k^{2}\right)\right]$

where $s$ is the square of the center-of-mass energy and $f_{M}$ and $f_{N}$ are respectively the meson and nucleon form factors. The total cross section is related to this amplitude by

$$
\sigma_{T}=\frac{\operatorname{Im} A(s, t=0)}{s}
$$

and, for simplicity, we will use a form factor in the pole approximation:

$$
f_{i}\left(k^{2}\right)=\frac{1}{\left(1+\frac{\left\langle r_{i}^{2}\right\rangle}{6} k^{2}\right)}
$$

We previously remarked that the leading two-gluon exchange cannot describe the growth with energy of the cross section. We compute the coefficient of the term $s^{0.08}$ in the total cross section. For example, a recent Regge fit for the $\pi^{-} p$ and $K^{-} p$ total cross sections [15] gives

$$
\begin{aligned}
& \pi^{-} p: \quad 13.63 s^{0.0808}+36.02 s^{-0.4525} \\
& K^{-} p: 11.82 s^{0.0808}+26.36 s^{-0.4525}
\end{aligned}
$$

Our model is expected to accommodate the values 13.63 and $\mathbf{1 1 . 8 2}$ in the above fit. Computing the ratio of cross sections we expect a factor of approximately $1 / 3$ for the $s^{0.0808}$ coefficients of $\sigma_{\psi p} / \sigma_{\pi p}$. The total $J / \psi-p$ cross section is $4 \mathrm{mb}$.

The ratios of cross sections are functions of the propagators and form factors. We used the following mean squared radii [14]: $\left\langle r_{p}^{2}\right\rangle=0.67 \mathrm{fm}^{2},\left\langle r_{\pi}^{2}\right\rangle=0.44 \mathrm{fm}^{2}$, 


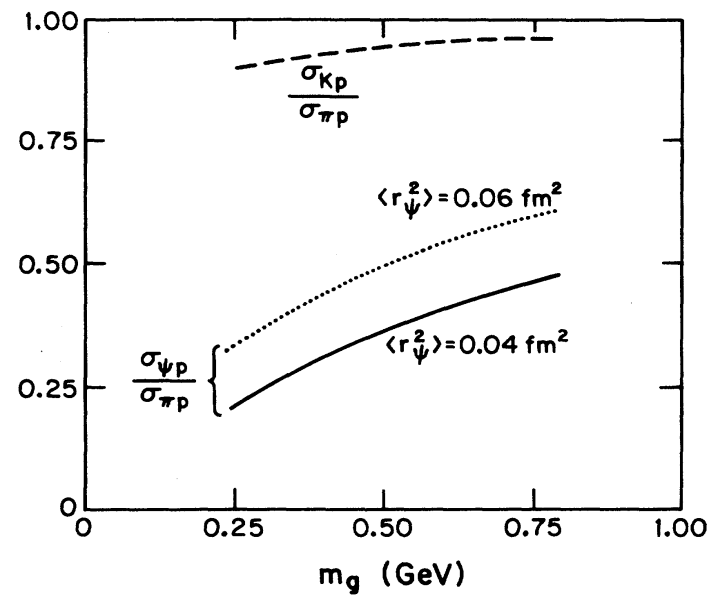

FIG. 3. Ratios of total cross sections: $\sigma_{K p} / \sigma_{\pi p}$ (dashed curve), $\sigma_{\psi p} / \sigma_{\pi p}$ with $\left\langle r_{\psi}^{2}\right\rangle=0.04 \mathrm{fm}^{2}$ (solid curve), and $\left\langle r_{\psi}^{2}\right\rangle=0.06 \mathrm{fm}^{2}$ (dotted curve). The curves were determined for $\Lambda=0.3 \mathrm{GeV}$.

$\left\langle r_{K}^{2}\right\rangle=0.35 \mathrm{fm}^{2},\left\langle r_{\psi}^{2}\right\rangle=0.04 \mathrm{fm}^{2}$, and also from a nonrelativistic quark model calculation $\left\langle r_{\psi}^{2}\right\rangle=0.06 \mathrm{fm}^{2}$. The ratios $\sigma_{K p} / \sigma_{\pi p}$ and $\sigma_{\psi p} / \sigma_{\pi p}$ given by Eqs. (10) and (11) are shown in Fig. 3 as a function of the gluon mass. Whereas the ratio $\sigma_{K p} / \sigma_{\pi p}$ is practically constant, $\sigma_{\psi p} / \sigma_{\pi p}$ exhibits an appreciable variation with $m_{g}$. This can be easily understood by recalling that, for a perturbative propagator with zero gluon mass and the form factor given by Eq. (12), the cross section dependence on hadron radius, e.g., for the collision of two identical mesons, is given by $[9,13]$

$$
\sigma_{M}=\frac{64}{27} \pi \alpha_{s}^{2}\left\langle r_{M}^{2}\right\rangle
$$

In this particular case $\left\langle r_{M}^{2}\right\rangle$ sets the scale of the cross section. When, however, $\left\langle r_{i}^{2}\right\rangle$ is too small (as in the case of $J / \psi)$ there is an interplay between this scale and $m_{g}$. The smaller $\left\langle r_{i}^{2}\right\rangle$ the stronger the dependence of the total cross section on the gluon mass. The curves of Fig. 3 are again obtained for $\Lambda=0.3 \mathrm{GeV}$. With $m_{g}=0.37 \mathrm{GeV}$ we have $\sigma_{K p} / \sigma_{\pi p} \simeq 0.92$ (to be compared with 0.87 ), and $\sigma_{\psi p} / \sigma_{\pi p} \simeq 0.29$ with $\left\langle r_{\psi}^{2}\right\rangle \simeq 0.04 \mathrm{fm}^{2}$. From Eq. (13) we predict the Pomeron contribution to $\sigma_{\psi p}$ to be equal to $3.95 s^{0.0808}$.

\section{CONCLUSIONS}

The LN model describes the Pomeron as the exchange of two nonperturbative gluons. In our study this nonperturbative gluon has a propagator given by a fit to the numerical solution of a gauge invariant set of the Schwinger-Dyson gluonic equations of QCD. At low $k^{2}$ the propagator shows the presence of a dynamically generated gluon mass, and at high $k^{2}$ has the correct QCD asymptotic behavior. In an earlier work we computed the elastic cross section for $p p$ scattering, finding agreement with experiment for $m_{g}=0.37 \mathrm{GeV}$ when $\Lambda=0.3 \mathrm{GeV}$. This value is also consistent with Cornwall's determination of $m_{g}$ through the gluon condensate. Here we computed the Pomeron coupling to quarks, the exclusive $\rho$ production in deep inelastic scattering, the ratio of total cross section of $J / \psi-p$ to $\pi-p$ scattering, and determined the behavior $3.95 s^{0.0808}$ for the Pomeron contribution to $J / \psi-p$ scattering. All the results are consistent with a gluon mass of $0.37 \mathrm{GeV}$ when $\Lambda_{\mathrm{QCD}}=0.3 \mathrm{GeV}$.

The fact that for hadrons with very small $\left\langle r_{i}^{2}\right\rangle$ the dependence on the gluon mass is stronger can possibly be tested experimentally. In our model there is no longer a direct relationship between total cross sections and hadronic radii [14].

The advantage of our scheme is that it is based on $\mathrm{QCD}$, and once $\Lambda_{\mathrm{QCD}}$ is precisely determined, the gluon mass is the only free parameter. The value of the strong coupling constant at low energy is fixed for consistency with the gluon propagator solution. No ad hoc choice of $g$ is necessary. Finally, all the processes studied up to now are consistent with a unique value for the gluon mass.

\section{ACKNOWLEDGMENTS}

This research was supported in part by the University of Wisconsin Research Committee with funds granted by the Wisconsin Alumni Research Foundation, by the U.S. Department of Energy under Contract No. DEAC02-76ER00881, by the Texas National Research Laboratory Commission under Grant No. RGFY9273, and by the Conselho Nacional de Desenvolvimento Cientifico e Tecnologico, CNPq (Brazil).
[1] F. E. Low, Phys. Rev. D 12, 163 (1975); S. Nussinov, Phys. Rev. Lett. 34, 1268 (1975).

[2] P. V. Landshoff and O. Nachtmann, Z. Phys. C 35, 405 (1987).

[3] J. M. Cornwall, Phys. Rev. D 26, 1453 (1982).

[4] F. Halzen, G. Krein, and A. A. Natale, Phys. Rev. D 47, 295 (1993).

[5] S. Mandelstam, Phys. Rev. D 20, 3223 (1979); M. Baker, J. S. Ball, and F. Zachariasen, Nucl. Phys. B186, 531 (1981); B186, 560 (1981); N. Brown and M. R. Pennington, Phys. Rev. D 38, 2266 (1988); 39, 2723 (1989);
J. R. Cudell and D. A. Ross, Nucl. Phys. B359, 247 (1991).

[6] C. Bernard, Phys. Lett. 108B, 431 (1982); P. A. Amundsen and J. Greensite, Phys. Lett. B 173, 179 (1986); J. E. Mandula and M. Ogilvie, ibid. 185, 127 (1987).

[7] A. Donnachie and P. V. Landshoff, Phys. Lett. B 185, 403 (1987); Nucl. Phys. B311, 509 (1988/89).

[8] J. R. Cudell, Nucl. Phys. B336, 1 (1990).

[9] J. Dolejsi and J. Hufner, Z. Phys. C 54, 489 (1992).

[10] G. Parisi and R. Petronzio, Phys. Lett. 94B, 51 (1980).

[11] J. M. Cornwall, in Deeper Pathways in High-Energy 
Physics, edited by B. Kursunoglu, A. Perlmutter, and L. Scott (Plenum, New York, 1977), p. 683; Nucl. Phys. B157, 392 (1979).

[12] Some authors have presented arguments that in the ultraviolet regime the running mass should fall off faster as $m^{2}\left(k^{2}\right)$; see J. M. Cornwall and Wei-Shu Hou, Phys. Rev. D 34, 585 (1986); M. Lavelle, ibid. 44, R26 (1991). The ultraviolet behavior does not affect our results significantly as Pomeron exchange is characterized by the infrared behavior of the propagator.
[13] J. F. Gunion and D. E. Soper, Phys. Rev. D 15, 2617 (1977); E. M. Levin and M. G. Ryskin, Yad. Fiz. 41, 1622 (1985) [Sov. J. Nucl. Phys. 41, 1027 (1985)].

[14] B. Povh and J. Hufner, Phys. Rev. Lett. 58, 1612 (1987); Phys. Lett. B 245, 653 (1990).

[15] A. Donnachie and P. V. Landshoff, Phys. Lett. B 296, 227 (1992).

[16] EMC Collaboration, J. J. Aubert et al., Phys. Lett. 161B, 203 (1987). 